\title{
Short-term Outcomes of Herbert Screw Fixation for Isolated Olecranon Fractures in Children: a Single- institution Retrospective Study
}

Yang Li

Shandong University Qilu Hospital https://orcid.org/0000-0002-7586-0190

Kelai Wang

Shandong University Qilu Hospital

Dong Sun

Shandong University Qilu Hospital

Yakun Liu

Shandong University Qilu Hospital

Jingwei Liu

Shandong University Qilu Hospital

\section{Zhe Wang}

Shandong University Qilu Hospital

Aiwu Li ( $\sim$ liaw0613@163.com )

Shandong University Qilu Hospital

\section{Research Article}

Keywords: isolated olecranon fractures, Herbert screw fixation, children

Posted Date: March 18th, 2021

DOl: https://doi.org/10.21203/rs.3.rs-287204/v1

License: (9) This work is licensed under a Creative Commons Attribution 4.0 International License.

Read Full License

Version of Record: A version of this preprint was published at Indian Journal of Orthopaedics on February 18th, 2022. See the published version at https://doi.org/10.1007/s43465-022-00609-4. 


\section{Abstract}

Background: Although various fixation methods can be used for the treatment of displaced olecranon fractures, there are no clear indications in the current literature regarding which surgical technique should be adopted. In this study, we evaluated the clinical and radiological outcomes of closed reduction with percutaneous Herbert screw fixation in children with isolated olecranon fractures.

Methods: We retrospectively reviewed the records of children treated at our center for isolated olecranon fractures (Mayo type IIA) with closed reduction and percutaneous Herbert screw fixation between January 2016 and December 2018. Radiographic assessment of fracture healing was performed 6-8 months postoperatively and included assessment for loss of reduction and maximum length of the ulna. Clinical outcomes included elbow flexion and extension, forearm pronation and supination, short version of the Disabilities of the Arm, Shoulder, and Hand (QuickDASH) score, and complications. The Herbert screws were removed by a second operation.

Results: A total of 14 patients with an average age at the time of injury of 11.36 (range, 10-14) years were included. All patients had good radiological and clinical outcomes at 6-8 months postoperatively; all had normal elbow ranges of motion and showed complete bone healing on radiographs. There were no cases of foreign body irritation, implant migration, or osteoarthritis. Premature epiphyseal closure was noted in six patients. The average QuickDASH score was 1.58.

Conclusions: Fixation of olecranon fractures with Herbert screws is a safe and easy fixation method in young patients, leading to good functional and radiological results. Nonetheless, determination of the effects of this treatment method on the olecranon ossification center requires long-term follow-up.

\section{Background}

Elbow fractures are common in children, accounting for $5-10 \%$ of all pediatric fractures. Contrarily, only $1.7 \%$ of healthy children are likely to have olecranon fracture[1]. Pediatric patients are likely to have injuries related to a high-energy injury mechanism. Olecranon fractures may occur when the elbow is extended or flexed by external forces, with a varus or valgus stress on the joint. The posterior surface of the olecranon is mainly affected by the intense extension of the triceps brachii muscle and the flexural stress generated by the biceps brachii muscle, which often lead to olecranon fractures[2].The anatomic site and pattern of the fracture, extensor mechanism integrity, and in particular, fracture displacement and stability, are the determining factors in the treatment of olecranon fractures.

Most olecranon fractures in children can be treated conservatively with good results. Fractures with a displacement of less than $2 \mathrm{~mm}$ are typically treated with cast immobilization, and long-term follow-up is performed to ensure good results [1,3]. Conversely, fractures with a displacement of more than $4 \mathrm{~mm}$ are treated surgically. Evans and Graham suggested that displacements of 2-4 mm represent a gray zone in which the choice for surgical or non-surgical treatment has to be individually considered depending on the biomechanical stability, as assessed clinically by an experienced surgeon [4]. 
Numerous classification systems have been described in the literature for olecranon fractures, but the most commonly used is the Mayo classification. Although various fixation methods can be used for the treatment of displaced olecranon fractures, such as isolated pinning, percutaneous screw fixation, cerclage with Kirschner wires, pinning with threaded pins with adjustable lock, and tension band wire (TBW) fixation $[1,5,6]$, there are no clear indications in the current literature regarding which surgical technique should be adopted for the treatment of Mayo type IIA olecranon fractures. The most commonly used surgical techniques for simple olecranon fractures are TBW and tension band suture (TBS) fixation, which produce good outcomes with union occurring in nearly $90 \%$ of fractures with a single surgical procedure $[3,7]$. However, these techniques are associated with many complications that are directly or indirectly correlated with the use of Kirschner wires, such as hardware irritation, Kirschner wire migration, and high reoperation rates for implant removal in a substantial proportion of patients (approximately $80 \%$ ) [8]. Furthermore, the open reduction and extensive exposure increases the risk for ischemic insult of the growth plate [9].

In 1942, MacAusland described the treatment of olecranon fractures by intramedullary screw fixation, but subsequent reports noted that the technique was challenging and unreliable [10-11]. Persiani et al. reported the outcomes of treatment of olecranon fractures in children affected with osteogenesis imperfecta $(\mathrm{OI})$ type I by screw fixation. Although screws provided good fixation, there was a high risk for implant migration; dispalcement of the internal fixation device occurred in the three of the 10 patients [12]. However, limited studies have reported on the use of screws for isolated olecranon fractures in healthy children and skeletally immature patients. Hence, the indications and type of surgical treatment for these fractures in these populations are not clear.

Thus, the purpose of this study was to assess the clinical and functional outcomes of pediatric isolated Mayo IIA type olecranon fractures treated with Herbert screws using the short version of the Disabilities of the Arm, Shoulder, and Hand (QuickDASH) outcome questionnaire and imaging examination.

\section{Methods}

\section{Study design and patients}

We retrospectively reviewed the records of children treated at the Qilu Hospital of Shandong University for olecranon fractures by closed reduction with percutaneous Herbert screw fixation from January 2016 to December 2018. The inclusion criteria were as follows: 1) Mayo type IIA isolated and closed olecranon fracture, 2) displacement $>4 \mathrm{~mm}, 3$ ) no neurovascular injury, and 4) age between 10 and 14 years. The exclusion criteria were as follows: 1) extra-articular ulnar fractures, 2) combined forearm fractures, 3 ) comminuted olecranon fractures, 4) conservative or TBW or TBS treatment, 4) age $<10$ or $>14$ years, and 5) non-visible olecranon ossification center or visible epiphyseal closure on radiographs. The following data were collected: patient's age, sex, mechanism of injury, operation time, side involved, length of postoperative immobilization, and contact information.

\section{Diagnosis and preoperative evaluation}


All patients were initially evaluated in the emergency room. At first contact with the patient, if olecranon fracture was suspected, X-ray examination of the elbow was performed. If olecranon fracture was confirmed, a pediatric orthopedic surgeon performed temporary plaster fixation in the emergency room to avoid aggravation of the fracture displacement. Patients with no surgical contraindications were hospitalized and underwent closed reduction and Herbert screw fixation in the operating room. Figure 1 shows representative preoperative images of a 13-year-old boy. Figure 2 shows postoperative images of the boy.

\section{Surgical technique}

Surgery was performed by an experienced pediatric orthopedic surgeon. After anesthesia induction, the patient was placed in the supine position, and the affected limb was placed on a C-arm X-ray device and sterilized. Because of the extension force of the triceps tendon, the fracture was generally displaced proximally and posteriorly; thus, we generally needed to keep the elbow straight. In some patients, fracture reduction was difficult to perform, and we needed to temporarily place a Kirshner wire into the proximal fracture end and use it to pry the reduction. After fracture reduction, we pressed the fracture block with fingers or a Kirshner wire and implanted two 0.8-mm Kirshner guide wires from the proximal to the distal end. Due to the fracture angle, it was difficult to pass the Kirshner wires through the proximal ulnar bone cortex. Following the direction of the Kirshner guide wires, we implanted two Herbert screws with a diameter of $3.0 \mathrm{~mm}$ and withdrew the Kirshner wires. The elbow joint was moved to detect the presence of obstructive sensation or limitation in joint movement. Strong fixation of the olecranon fracture was observed. If the fracture was confirmed to be in good reduction during the surgery-firmly fixed fracture end, no displacement of the internal fixation device, straight elbow joint with normal flexion activity-the forearm was placed in the functional position with the elbow joint flexed at 45 degrees and fixed with polyester bandage.

\section{Postoperative course and follow-up}

All patients were under observation after the surgery to monitor the blood supply in the fingers. In two patients, inlay pressure of the plaster was observed in the early stage, and the plaster was opened and decompressed in the ward. Neither of the two patients showed symptoms of osteofascial compartment syndrome in the later stage. Penicillin was used pre- and postoperatively to prevent infection.

Outpatient follow-ups and radiographic examinations were performed in all patients 1,3 , and 6 months after surgery. On the first postoperative visit 4 weeks after surgery, if the fracture line was blurred, we removed the cast and prompted functional activity. If the fracture line was clearly visible, cast removal was delayed until 6 weeks after the surgery. Three of the patients had less callus at the fracture site 4 weeks after surgery, and we delayed the cast removal by 2 weeks. Exercise was assisted by family members or a rehabilitation physician. At the 6-month follow-up, we used the QuickDASH to assess the elbow joint function and recorded forearm muscle strength, elbow flexion and extension range, and bilateral maximum length of the ulna (Fig. 3 ). 
Owing to the concern that cannulated compression screws may affect the growth of the epiphyseal or secondary ossification centers, removal of internal fixation was performed in most patients between 6 and 8 months after surgery(Fig. 4). Radiological comparison between the fractured and non-fractured arm to evaluate the effert of herbert screw on the epiphyseal and measurement the maximum length of the ulna(Fig. 5). Relevant information is still being followed up.

\section{Statistical analysis}

All analyses were performed using IBM SPSS Statistics for Windows, version 19.0 (IBM Corp., Armonk, NY, USA). Data were expressed as means \pm standard deviations. Comparison between the fractured and non-fractured arms in the same individuals was made using the paired t-test. The threshold for statistical significance was set at $p<0.05$.

\section{Results}

A total of 14 patients were included in the study. There were 9 males and 5 females, with an average age at the time of injury of 11.36 (range, 10-14) years. All fractures were closed injuries. None of the patients required open reduction. The average follow-up time was 11.9 (range, 8-15) months and the average QuickDASH score was 1.58 (Table 1).

Table 1

Patients' characteristics

\begin{tabular}{|lllll|}
\hline Number & $\begin{array}{c}\text { Age } \\
(\mathrm{yr})\end{array}$ & $\begin{array}{l}\text { Surgery } \\
\text { duration } \\
(\mathrm{min})\end{array}$ & $\begin{array}{l}\text { Number of intraoperative fluoroscopy } \\
\text { procedures }\end{array}$ & $\begin{array}{l}\text { QuickDASH } \\
\text { score }\end{array}$ \\
\hline 14 & 11.36 & 31.7 & 36.5 & 1.58 \\
\hline
\end{tabular}

Cast removal was performed at 28 days after surgery in 11 patients, while three patients had their plaster removed at 6 weeks after surgery because of lack of callus growth. X-ray imaging showed that all patients had complete bone healing, and there were no cases of periarticular calcification, foreign body irritation, persistent joint pain, or implant migration. At the 6-month follow-up, we measured the elbow ranges of motion and the maximum length of the ulna both on the fractured and non-fractured arms (Table 2). In the comparison, there was no significant difference between the fractured and non-fractured arms. Internal fixation was removed in all patients 6 to 8 months after surgery. Radiographs were also obtained from the uninjured side to compare the development of the olecranon epiphyseal cartilage. There were six patients (42.9\%) with premature epiphyseal closure. 
Table 2

Comparison of clinical outcomes between the fractured and non-fractured arms

\begin{tabular}{|lllll|}
\hline & Fractured arm & Non-fractured arm & t & p \\
\hline Elbow flexion $\left(^{\circ}\right)$ & $143.93 \pm 1.38$ & $14516 \pm 2.21$ & 1.589 & 0.136 \\
\hline Elbow extension $\left(^{\circ}\right)$ & $1.80 \pm 0.89$ & $1.17 \pm 0.60$ & 2.080 & 0.058 \\
\hline Forearm pronation $\left(^{\circ}\right)$ & $84.14 \pm 1.28$ & $85.10 \pm 1.73$ & 1.445 & 0.172 \\
\hline Forearm supination $\left(^{\circ}\right)$ & $85.71 \pm 3.26$ & $88.02 \pm 2.22$ & 2.138 & 0.052 \\
\hline Maximum length of the ulna $(\mathrm{cm})$ & $22.89 \pm 2.80$ & $22.91 \pm 1.51$ & 0.017 & 0.987 \\
\hline Data are presented as means \pm standard deviations. $\mathrm{p}<0.05$ was considered significant. \\
\hline
\end{tabular}

\section{Discussion}

In this study, our results indicated that fixation of olecranon fractures with Herbert screws is a safe and easy fixation method in young patients, leading to good functional and radiological results.

Olecranon fractures are rare in children, representing approximately $5 \%$ of all elbow fractures [6]. Because these fractures involve the articular surface of the elbow joint and may affect the development of ulnar bone epiphyses, the standard indication for surgical reduction is generally considered to be a fracture displacement distance greater than $4 \mathrm{~mm}$ [13].

In previous studies, TBW and TBS have been described as very effective fixation methods with excellent results, even regarded as the gold standard for simple olecranon fractures [14]. However, because the skin at the proximal ulna is thin with relatively little subcutaneous tissue, although the tension bands maintain a good and stable reduction at the olecranon fracture site, hardware irritation or persistent joint pain often develop, requiring hardware removal in up to $68-82 \%$ of patients [14]. The need for additional surgery is mostly determined by intolerance to the osteosynthesis material due to the prominent Kirschner wires in the elbow [15-16]. When hollow screws are used for internal fixation, the end of the screws is buried in the ulnar periosteum and foreign body rejection occurs rarely. Furthermore, soft tissue irritation by the screws during elbow movement can be negligible. In this study, none of the 14 patients experienced hardware irritation or movement obstruction during elbow flexion or extension.

Good reduction and stable fixation are critical for the recovery of olecranon fractures. Because these fractures involve the ulnar articular surface, it is required that the fracture gap during surgery be less than $2 \mathrm{~mm}$. In Herbert screw fixation, the screws are pressurized by the head and tail thread difference and the number of screw-in threads to reduce the fracture gap and achieve a better reduction. Although it has been shown that $39-55 \%$ of compression is lost during the first $12 \mathrm{~h}$ after fixation, good initial compression remains important [17]. 
The triceps tendon, which is attached to the olecranon, will pull the olecranon back after fracture reduction. It has been reported that there is no difference in the reoperation and complication rates between TBW and screw fixation in adult olecranon fracture [18]. However, it should be noted that in patients with OI type I, there is a risk of loose internal fixation, which can be attributed to an inadequate adhesion of the implant to the osteoporotic bone in these patients [12]. In our study, the elbow joint was straightened and flexed after the Herbert screws were implanted to assess the stability of the cannulated screws. There were no cases of implant migration, although some screws did not penetrate the distal cortical bone. This indicates that the Herbert screw can provide sufficient holding force to resist the pull of the triceps.

In addition, elbow movement was restricted in all patients after surgery with a tubular plaster cast. The plaster cast was applied with the elbow at 45 degrees flexion and the triceps in a relaxed state, minimizing the traction force to the olecranon. Immobilization has been associated with a reduced incidence of internal fixation displacement and revision. Good primary compression and external fixation ensures fracture healing by eliminating the possibility of internal fixation displacement [19].

The use of TBW fixation requires open reduction. In this study, none of the patients required open reduction. In all patients, reduction was completed under fluoroscopy with Kirshner wires. Compared to TBW, Herbert screw fixation is a better choice because of the smaller incision with minimal soft-tissue dissection and trauma.

The growth plate activity in the upper extremity was quantitatively investigated by Pritchett, who reported that $15 \%$ of the overall ulnar growth occurs at the proximal growth plate; the growth decreases to $5 \%$ by the age of 8 years [20]. The ulnar epiphyseal ossification center, mainly involved in the development of the proximal ulnar bone, appears at the age of 9-12 years and closes at the age of 14-17 years. Although it contributes to a very small percentage of the total longitudinal ulnar growth, disruption of the olecranon growth plate by means of fractures, infections, or surgical interventions may cause deformity and/or long-term prospective growth arrest of the forearm.

The diameter of the internal fixation screws in this study was $3.0 \mathrm{~mm}$, and there was a risk of implantinduced growth arrest and formation of a bony bridge. However, considering the age of the patients in this study and the contribution of the olecranon epiphysis to ulnar development, the influence was considered to be minimal. In their study on the outcomes of intramedullary nail fixation through the olecranon apophysis in skeletally immature forearm fractures, Rabinovich et al. found no significant ulnar length disruption or functional limitations [20]. During the follow-up, we found that none of the patients had unequal limb length, although six patients had premature epiphyseal closure. Therefore, it can be said that growth arrest caused by the insertion of hollow screws is very rare and insignificant, particularly considering that secondary ossification centers of the olecranon had emerged in our patients.

There are several limitations to our study that relate to its retrospective design. First, this was a singleinstitution study and the treatment outcome depended on a single surgeon. Hence, this study has low representativeness. Second, our sample size was small, and the follow-up period was short. Therefore, we 
could not determine the impact of Herbert screws on the epiphyses. However, we will continue to follow up these patients, and relevant data will be collected and analyzed.

\section{Conclusions}

In the present study, we found no significant contraindications for the use of Herbert screws in patients aged 10-14 years. None of the patients developed osteoarthritis during the short-term follow-up.

Furthermore, the QuickDASH score for the elbow was inspiring. Compared with TBW and TBS, Herbert screw fixation is a reasonable treatment option for olecranon fractures that can significantly reduce the incidence of persistent joint pain, implant migration, and particularly, foreign body irritation. Furthermore, the QuickDASH score for the elbow was inspiring.

\section{List Of Abbreviations}

Ol, osteogenesis imperfecta;

QuickDASH, short version of the Disabilities of the Arm, Shoulder, and Hand questionnaire;

TBS, tension band suture;

TBW, tension band wire.

\section{Declarations}

\section{Ethics approval and consent to participate}

The study was approved by the Medical Ethics Committee of Qilu Hospital of Shandong University (approval number KYLL-2020008-165). All study procedures were in line with the tenets of the 1964 Declaration of Helsinki and its later amendments or comparable ethical standards.

\section{Consent for publication}

Not applicable.

\section{Availability of data and materials}

The datasets used and/or analyzed during the current study are available from the corresponding author on reasonable request.

\section{Competing interests}

The authors declare that they have no competing interests.

\section{Funding}


This research was supported by the Natural Science Foundation of Shandong Province (grant No. ZR2020GSF118021). The funders had no role in the study design, data collection and analysis, decision to publish, or preparation of the manuscript.

\section{Authors' contributions}

LY contributed to study conception and data collection, performed the surgeries;WKL follow-up assessments, and drafted the manuscript; SD and LYK performed the radiologic analysis and literature review; LJW contributed to data collection, WZ contributed to the follow-up assessments. LAW supervised the study and are guarantors for the accuracy of the study data. All authors have read and approved the submitted manuscript.

\section{References}

1. Caterini R, Farsetti P, D'Arrigo C, Ippolito E. Fractures of the olecranon in children. Long-term follow-up of 39 cases. J Pediatr Orthop B. 2002 Oct;11(4):320-8.

2. Kalbitz, M., Weber, B., Lackner, I. et al. Olecranon fractures in children: treatment of a rare entity. Eur J Trauma Emerg Surg (2020).

3. Chalidis BE, Sachinis NC, Samoladas EP, Dimitriou CG, Pournaras JD. Is tension band wiring technique the "gold standard" for the treatment of olecranon fractures? A long term functional outcome study. J Orthop Surg Res 2008;3:9.

4. Evans MC, Graham HK. Olecranon fractures in children: Part 1: a clinical review; Part 2: a new classification and management algorithm. J PediatrOrthop. 1999;19(5):559-69.

5. den Hamer A, Heusinkveld M, Traa W, Oomen P, Oliva F, Del Buono A, Maffulli N (2015) Current techniques for management of transverse displaced olecranon fractures. Muscles Ligaments Tendons J 5:129-140

6. C A Perkins, M T Busch \et al Olecranon fractures in children and adolescents: outcomes based on fracture fixation J Child Orthop. 2018 Oct 1;12(5):497-501.

7. Mauffrey CP, Krikler S. Surgical techniques: how I do it? Open reduction and tension band wiring of olecranon fractures. Injury 2009;40:461-5.

8. Lu QF, Tang GL, Zhao XJ, Zhang WJ, Guo SG, Wang HZ. Tension band wiring through doublecannulated screws as a new internal fixation method for treatment of olecranon fractures: a randomized comparative study. Acta Orthop Traumatol Turc. 2015;49(6):654-60.

9. Arbes, S., Platzer, P. \& Vécsei, V. Surgical treatment of olecranon fractures in children. Eur J Orthop Surg Traumatol 22, 209-212 (2012).

10. Macausland WR. The treatment of fractures of the olecranon by longitudinal screw or nail fixation. Ann Surg. 1942;116:293-6.

11. Bosman WPF, Emmink BL, Bhashyam AR, Houwert RM, Keizer J. Intramedullary screw fixation for simple displaced olecranon fractures. Eur J Trauma Emerg Surg. 2020 Feb;46(1):83-89. 
12. Persiani P, Ranaldi FM, Graci J, De Cristo C, Zambrano A, D'Eufemia P, Martini L, Villani C. Isolated olecranon fractures in children affected by osteogenesis imperfecta type I treated with single screw or tension band wiring system: Outcomes and pitfalls in relation to bone mineral density. Medicine (Baltimore). 2017 May;96(20):e6766.

13. Karlsson MK, Hasserius R, Karlsson C, Besjakov J, Josefsson PO. Fractures of the olecranon during growth: a 15-25-year follow-up. J Pediatr Orthop B. 2002 Jul;11(3):251-5.

14. Ruben García-Elvira, Maria Antonia Vives-Barquiel Pilar Camacho-Carrasco.Olecranon mayo IIA fractures treated with transosseous high strength suture: series of 29 cases Injury 51 (2020) S94S102

15. Duckworth AD, Clement ND, Aitken SA, Court-Brown CM, McQueen MM. The epidemiology of fractures of the proximal ulna. Injury. 2012;43:343-6.

16. Snoddy MC, Lang MF, An TJ, Mitchell PM, Grantham WJ, Hooe BS, et al. Olecranon fractures: factors influencing re-operation. Int Orthop. 2014;38:1711-6.

17. Gruszka DS, Burkhart KJ, Nowak TE, Achenbach T, Rommens PM, Muller LP (2012) The durability of the intrascaphoid compression of headless compression screws: in vitro study. J Hand Surg Am 37(6):1142-1150

18. Claessen FM, Braun Y, Peters RM, Dyer G, Doornberg JN,Factors Ring D (2016) Associated with reoperation after fifixation of displaced olecranon fractures. Clin Orthop Relat Res 474(1):193-200

19. Corradin, M., Marengo, L., Andreacchio, A. et al. Outcome of isolated olecranon fractures in skeletally immature patients: comparison of open reduction and tension band wiring fixation versus closed reduction and percutaneous screw fixation. Eur J Orthop Surg Traumatol 26, 469-476 (2016).

20. Rabinovich, Alexander , A. Adili , and J. Mah . "Outcomes of intramedullary nail fixation through the olecranon apophysis in skeletally immature forearm fractures. " J Pediatr Orthop25.5(2005):565-569

\section{Figures}



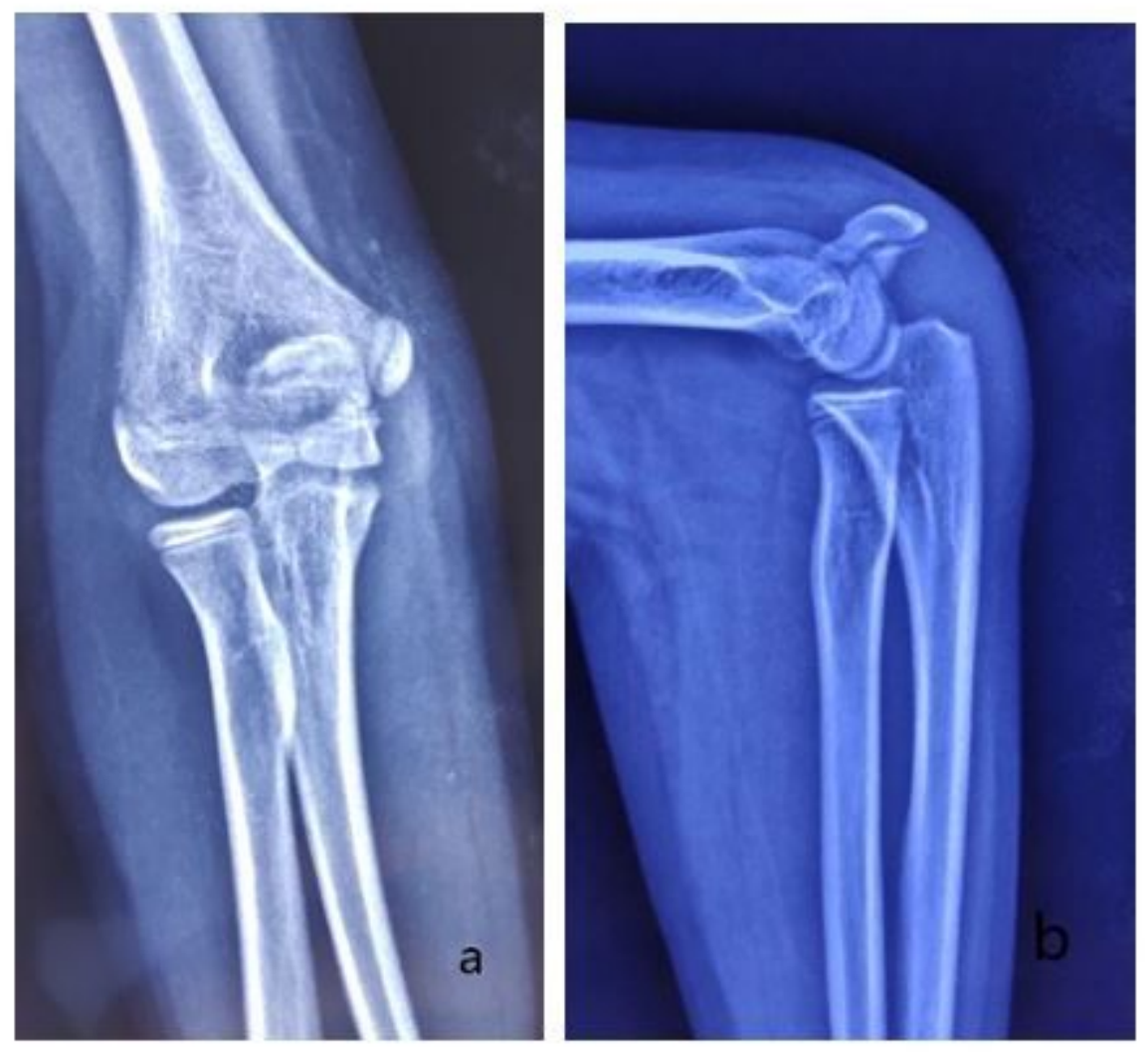

\section{Figure 1}

Preoperative images. (a) AP and (b) lateral radiographs in a 13-year-old boy with olecranon fracture.
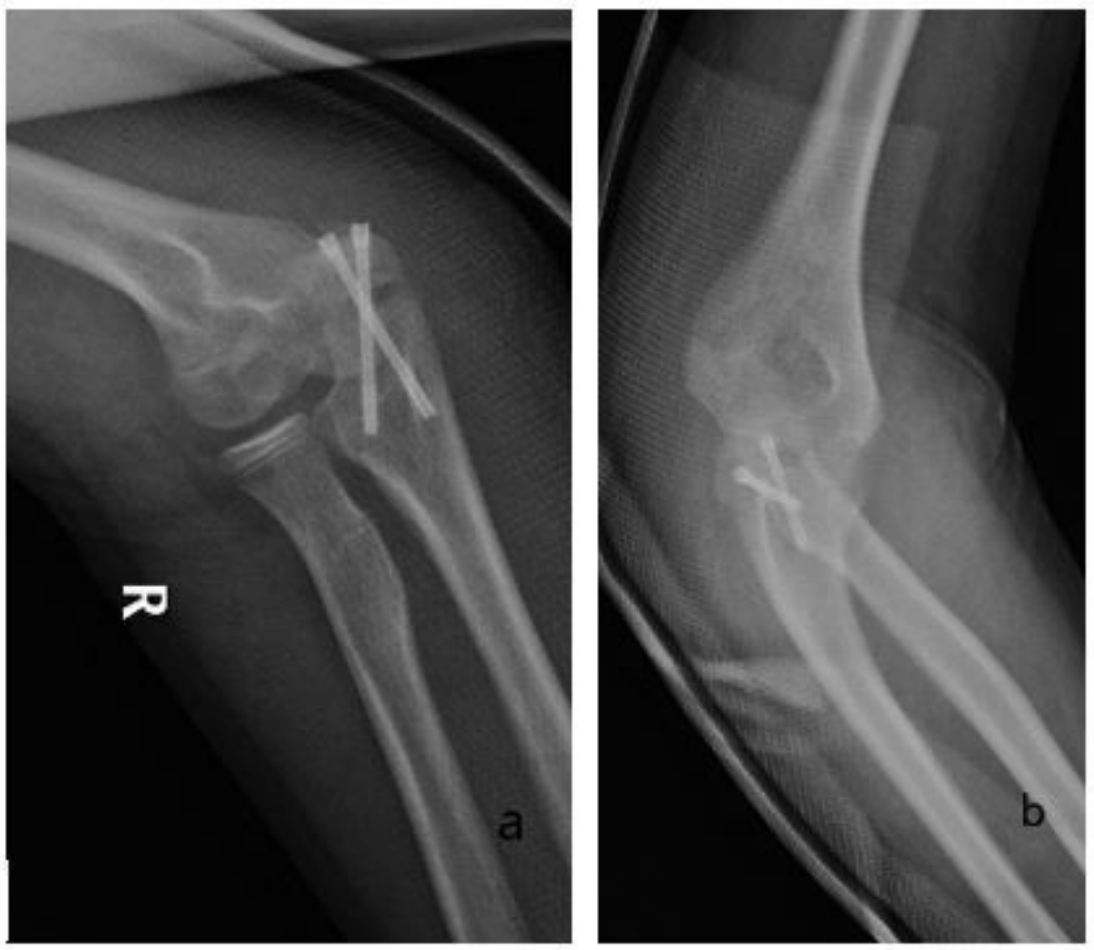

Figure 2 
Postopetative images from the 1-day follow up.(a) AP and (b) lateral radiographs in the same patient treated with closed reduction and Herbert screw fixation.
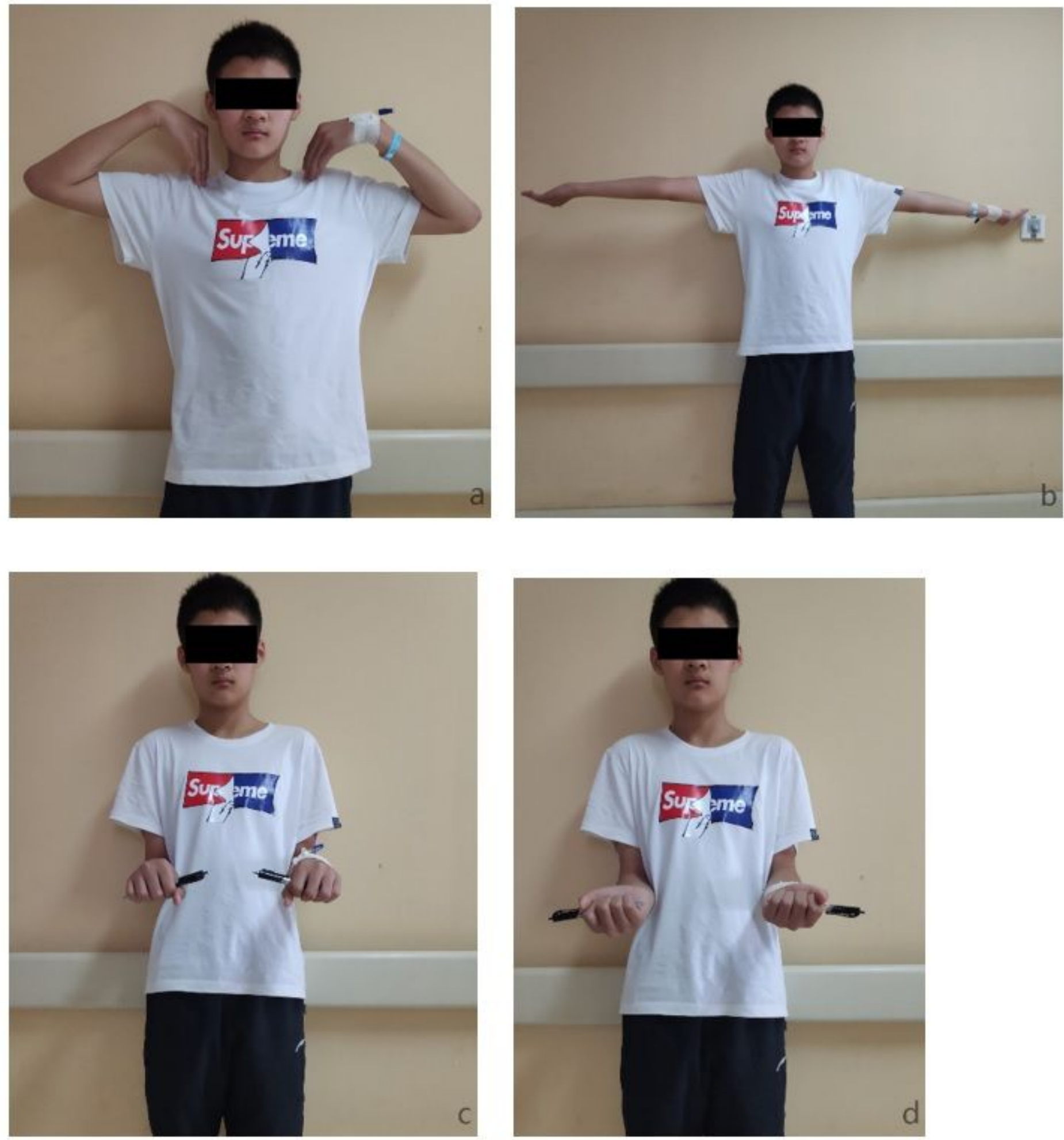

\section{Figure 3}

Functional outcomes in the same patient at 6 months after surgery. Before removing the cannulated screw, we recorded the patient's elbow ranges of motion in (a) flexion, (b) extension, (c) pronation, and (d) supination, the forearm length, and the QuickDASH score. 

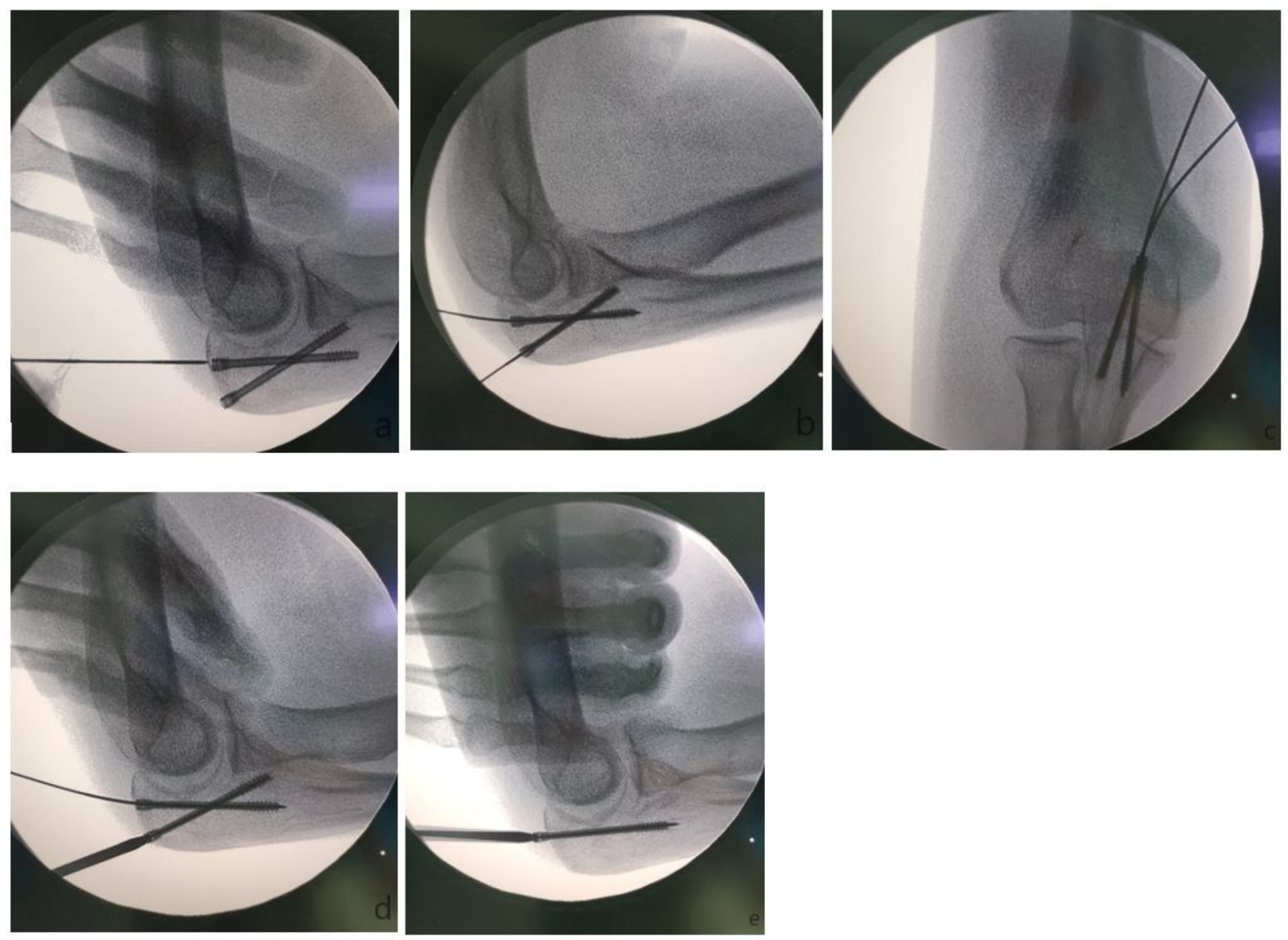

\section{Figure 4}

Hardware removal in the same patient 6 months after surgery. (a) A 0.8-mm Kirschner wire was inserted into the skin of the elbow and the Herbert screw. (b, c) AP and lateral radiographs showing that the Kirschner wire was inserted into the Herbert screw. (d, e) An incision was made in the skin around the Kirschner wire, and the Herbert screw was unscrewed in the direction of the Kirschner wire. 

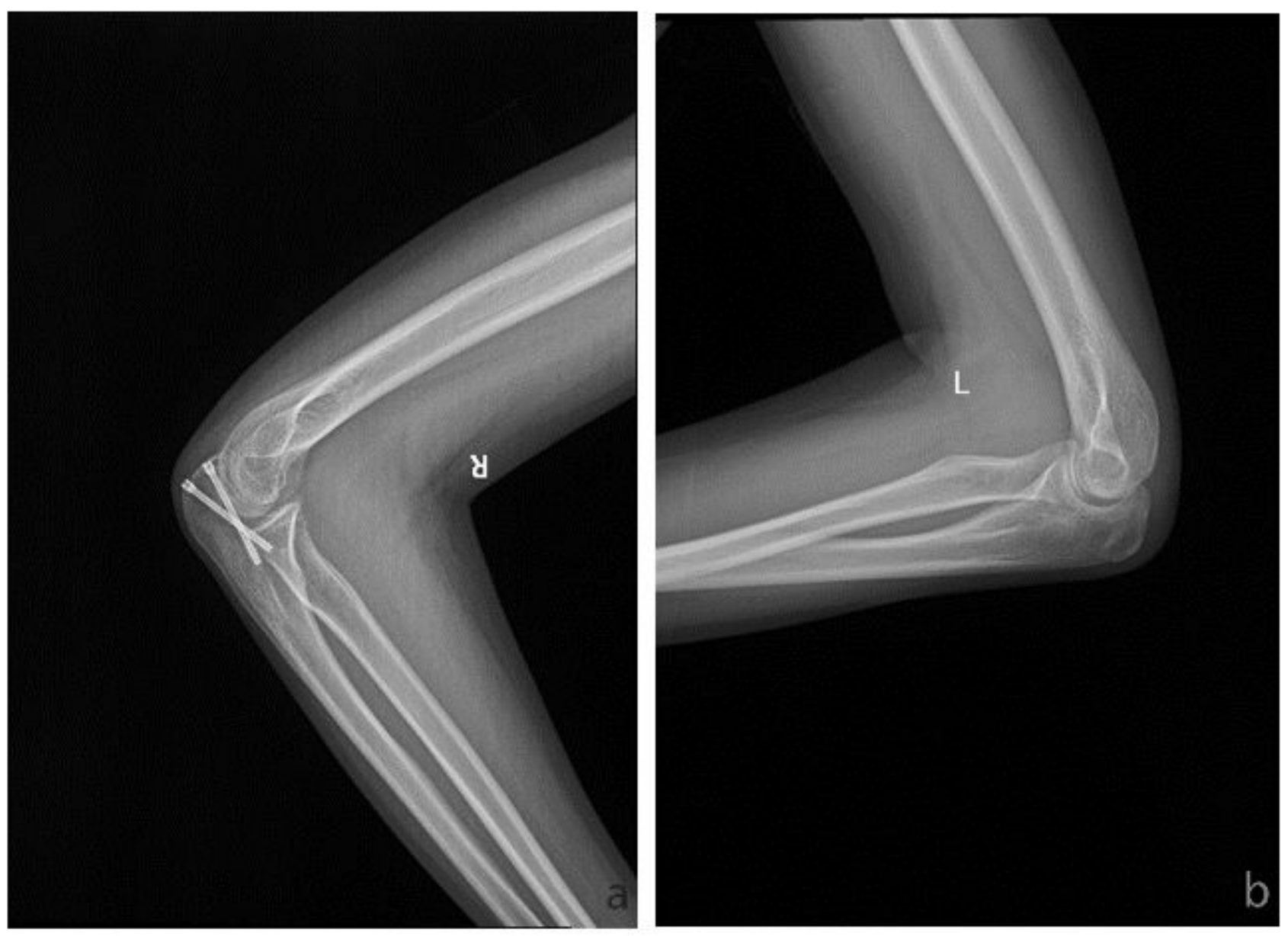

\section{Figure 5}

Follow-up images obtained at 6 months after surgery in the same patient. Radiological comparison between the fractured (a) and non-fractured (b) arms. 